\title{
Key populations for early COVID-19 immunization: preliminary guidance for policy
}

\author{
Shainoor J. Ismail MD MSc, Linlu Zhao PhD, Matthew C. Tunis PhD, Shelley L. Deeks MD MSc, \\ Caroline Quach MD MSc; for the National Advisory Committee on Immunization
}

Cite as: CMAJ 2020 November 30;192:E1620-32. doi: 10.1503/cmaj.202353; early-released November 3, 2020

CMAJ Podcasts: author interview at www.cmaj.ca/lookup/doi/10.1503/cmaj.202353/tab-related-content

This article is available in French at www.cmaj.ca/lookup/doi/10.1503/cmaj.202353-f

A larming levels of spread of severe acute respiratory syndrome coronavirus 2 (SARS-CoV-2) triggered the World Health Organization (WHO) to declare novel coronavirus disease 2019 (COVID-19) a pandemic on Mar. 11, 2020. ${ }^{1}$ The pandemic is ongoing and has caused substantial morbidity and death, as well as social and economic disruption worldwide. Although the risk varies among and within communities, the risk to Canadians remains high, with 191732 publicly reported confirmed and probable cases in Canada as of Oct. 15, 2020. ${ }^{2}$

The goal of Canada's pandemic response is to minimize serious illness and deaths while minimizing societal disruption as a result of the COVID-19 pandemic. ${ }^{3}$ Infection prevention and control measures such as physical distancing have been critical to slow the spread of COVID-19. ${ }^{4}$

Immunization with a safe and effective vaccine could hasten the containment and control of disease with reduced morbidity and mortality, as has been accomplished with other vaccinepreventable diseases for more than 50 years. ${ }^{5}$ Global efforts to develop a SARS-CoV-2 vaccine are progressing at an unprecedented pace ${ }^{6}$ and the Government of Canada is reviewing regulatory pathways to ensure rapid access to effective vaccines for Canadians without compromising vaccine safety. ${ }^{7}$ Initial supplies of prospective SARS-CoV-2 vaccines are not expected to be sufficient to meet the demand for immunization.

Phase 3 randomized controlled trials of several SARS-CoV-2 vaccine candidates included in advance purchase agreements with the Government of Canada are enrolling large numbers of participants ( $n \geq 30000$ in each trial) and are primarily looking at efficacy in preventing infection, biological markers of immunity and safety. ${ }^{8-11}$

Transparent, clear communication about SARS-CoV-2 vaccines and vaccine allocation decisions is important to establish and maintain trust and confidence in vaccines. Willingness among Canadians to receive a safe, effective COVID-19 vaccine has decreased over time. ${ }^{12-15}$ Vaccine hesitancy - the reluctance or refusal to be vaccinated despite the availability of vaccines - could limit the success of a COVID-19 immunization program, and the WHO deemed this one of the top 10 major public health threats in $2019 .{ }^{16}$ In general, receiving

\section{KEY POINTS}

- Immunization with a safe and effective vaccine could hasten control of the coronavirus disease 2019 (COVID-19) pandemic and minimize morbidity, death and societal disruption that have resulted from it.

- Canada's National Advisory Committee on Immunization (NACI) has developed preliminary recommendations for the efficient, effective and equitable allocation of safe, efficacious severe acute respiratory syndrome coronavirus 2 (SARS-CoV-2) vaccine(s) in the context of staggered arrival of vaccines.

- Key populations for early vaccination include those at high risk of severe illness and death from COVID-19; those most likely to transmit COVID-19 to those at high risk of severe illness and death from COVID-19 and workers essential to maintaining the COVID-19 response; those contributing to the maintenance of other essential services for the functioning of society; and those whose living or working conditions put them at elevated risk of infection and where infection could have disproportionate consequences, including Indigenous communities.

- Sequencing of key populations will be determined once more information about SARS-CoV-2 vaccine(s) becomes available; integration of equity, feasibility and acceptability considerations across all populations is critical for decisions regarding a COVID-19 immunization program.

- Transparent, clear communication about SARS-CoV-2 vaccines and vaccine allocation decisions to the public and health care providers is important to establish and maintain trust and confidence, and improve access to vaccines.

a recommendation from a health care provider is linked to increased vaccine acceptability, ${ }^{17}$ and a notable factor for health care providers to suggest a vaccine is whether or not it has been recommended by an expert committee. ${ }^{18}$

The objective of this guideline from Canada's National Advisory Committee on Immunization (NACI) is to provide preliminary recommendations to inform planning for the efficient, effective and equitable allocation of safe, efficacious SARS-CoV-2 vaccine(s) authorized for use in Canada in the context of staggered arrival of vaccines. 
To achieve this objective, this guideline identifies key populations for early COVID-19 immunization. Sequencing of key populations will be determined once more information about SARS-CoV-2 vaccines is available from late-phase clinical trial data. At the time of writing, it is too early to tell how these vaccines will perform in terms of efficacy and duration of protection. These factors will be reflected in NACl's vaccine-specific guideline, which will be developed when vaccine(s) become authorized for use in Canada.

\section{Scope}

$\mathrm{NACl}$ recommendations on key populations for early COVID-19 immunization apply to publicly funded immunization programs and not to individuals wishing to prevent COVID-19 with privately purchased vaccines. Although the target users for these recommendations are policy-makers (e.g., federal, provincial and territorial decision-makers for public health program planning), health care providers and the public are also users of NACI guidance.

Specific recommendations for SARS-CoV-2 vaccines in key populations will depend on as yet unknown factors such as vaccine efficacy in different populations, the epidemiologic context at the time vaccine(s) become(s) accessible and total vaccine supply. When this information is available, $\mathrm{NACl}$ will provide additional evidence-informed guidance on options for COVID-19 immunization, which will inform publicly funded immunization programs, as well as individuals and health care providers wishing to advise their patients on options for publicly funded SARS-CoV-2 vaccines. $\mathrm{NACl}$ does not provide advice on vaccine stockpiling, procurement or allocation of vaccine among provinces and territories.

\section{Recommendations}

Figure 1 summarizes NACl's preliminary recommendations on key populations for early COVID-19 immunization for public health program-level decision-making. The full guideline is available at www.canada.ca/naci.

\section{Recommendations on key populations}

Due to anticipated challenges with sufficient supply to vaccinate the entire at-risk population initially, NACl recommends that key populations in whom vaccine is deemed safe and effective, based on evidence available at the time of vaccine availability, should be prioritized for COVID-19 immunization in order to minimize serious illness and deaths as a result of the pandemic. These groups are not mutually exclusive and may overlap. A sequential approach cannot be determined until vaccine characteristics, results of clinical trials and the number of available doses are known (consensus recommendation).

Key populations for early COVID-19 immunization include those at high risk of severe illness and death from COVID-19; those most likely to transmit COVID-19 to those at high risk of severe illness and death from COVID-19 and workers essential to maintaining the COVID-19 response; those contributing to the maintenance of other essential services for the functioning of society; and those whose living or working conditions put them at elevated risk of infection and where infection could have disproportionate consequences, including Indigenous communities (consensus recommendation).

Sequencing of key populations and subprioritization within key populations has been and will continue to be based on a populationbased risk-benefit analysis, taking into consideration risk of exposure, risk of transmission to others, risk of severe illness and death, and the safety and effectiveness of vaccine(s) in key populations; vaccine characteristics and results of clinical trials; vaccine supply; and COVID-19 epidemic conditions when the vaccine(s) become(s) available (consensus recommendation).

Table 1 outlines evidence on potential biological and social risk factors for severe COVID-19, and Table 2 summarizes the evidence and rationale for the recommendations on key populations for early COVID-19 immunization.

Decisions regarding the sequencing of key populations and subprioritization within key populations will be developed through careful consideration of the best available evidence and awareness of knowledge gaps. Recommendations will be amended as necessary as the evidence evolves.

\section{Recommendations on principles to guide decision- making}

Efforts should be made to increase access to immunization services to reduce health inequities without further stigmatization or discrimination, and to engage systematically marginalized and racialized populations in immunization program planning (consensus recommendation).

Federal, provincial and territorial jurisdictions should begin planning for the implementation of a COVID-19 immunization program, including rapid monitoring of safety, effectiveness and coverage of vaccine(s) in different key populations, as well as effective and efficient immunization of populations in remote and isolated communities (consensus recommendation).

Efforts should be made to improve knowledge about the benefits of vaccines in general and of COVID-19 vaccine(s) specifically, once available, to address misinformation about immunization, and to communicate transparently about COVID-19 vaccine allocation decisions (consensus recommendation).

Decisions regarding a COVID-19 immunization program should integrate equity, feasibility and acceptability considerations across all key populations. Table 3 summarizes the evidence and rationale for the recommendations on guiding principles for decision-making on a COVID-19 immunization program.

\section{Key considerations}

In order to develop comprehensive, appropriate recommendations on immunization programs, $\mathrm{NACl}$ considers a number of factors. In addition to critically appraising evidence on burden of disease and vaccine characteristics such as safety, efficacy, immunogenicity and effectiveness, $\mathrm{NACl}$ uses a published, 
peer-reviewed framework and evidence-informed tools to ensure that issues related to ethics, equity, feasibility and acceptability are systematically assessed and integrated into its guidance. ${ }^{27}$ The definitions NACl has adopted for the application of this framework are summarized in Table 4. The NACI Secretariat applied the evidence-informed tools (Ethics Integrated Filters, Equity Matrix, Feasibility Matrix, Acceptability Matrix) to this guideline; these are available in the full document (www.canada.ca/naci).

$\mathrm{NACl}$ deliberated on available evidence on the spectrum of considerations and transparently summarized the rationale for each recommendation (Tables 2 and 3). These considerations, including evolving evidence on COVID-19 epidemiology and vaccines, will inform future decisions on prioritization and the COVID-19 immunization program. In the absence of late-phase clinical trial data on SARS-CoV-2 vaccine characteristics such as safety and efficacy, NACl's considerations of ethics, equity, feasibility and acceptability are summarized below.

\section{Ethics}

To support ethical deliberations and decision-making, $\mathrm{NACl}$ applied its Ethics Integrated Filters (Appendices B and C in the

\section{KEY POPULATIONS FOR}

\section{EARLY COVID-19 IMMUNIZATION*}

Those who are:

$\checkmark$ At high risk of
severe illness
and death from
COVID-19
- Advanced age
- High-risk health
conditions

Key populations

may overlap

\section{$\checkmark$ Most likely to transmit to those at high risk of severe illness and death from COVID-19 \\ $\checkmark$ Essential to maintaining the COVID-19 response}

- Health care and other workers providing care for patients with COVID-19 or long-term care residents and older adults in other congregate-care facilities

- Household contacts of those at high risk

\section{$\checkmark$ Contributing to the maintenance of essential services}

- Those who can't work virtually (e.g., police, firefighters)

- To be defined and prioritized by governments

\section{$\checkmark$ At high risk of infection owing to living or working conditions and in settings where infection could lead to disproportionate consequences}

- Settings where physical distancing and other infection prevention and control measures are challenging and access to health care is reduced, including in Indigenous communities

\section{SEQUENCE AND SUBPRIORITIZATION WILL BE BASED ON:}

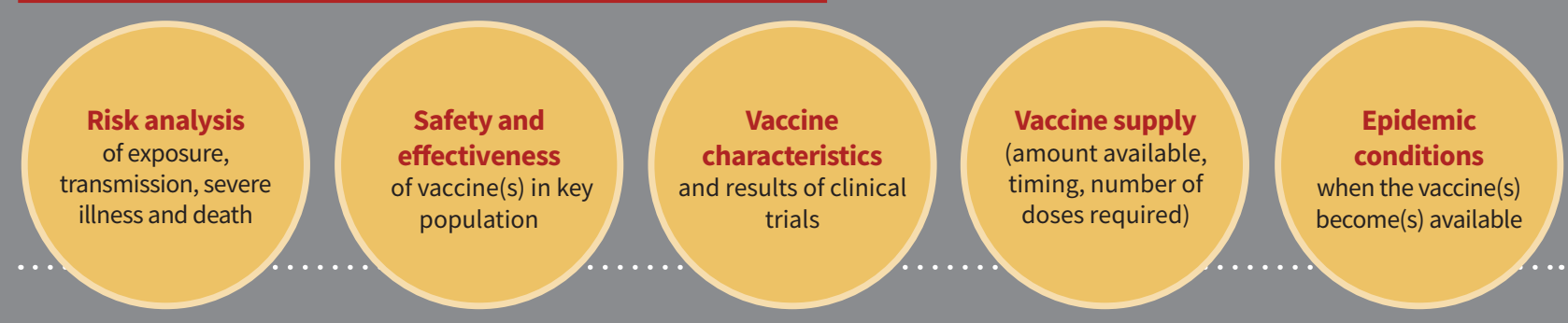

\section{PRINCIPLES TO GUIDE ETHICAL DECISION-MAKING: $†$}

\section{Equity}

Increase access to immunization services, reduce health inequities and stigmatization, engage marginalized and racialized groups

\section{Feasibility}

Plan for rapid monitoring of safety, effectiveness, vaccine coverage, access for remote or isolated communities

\section{Acceptability}

Improve knowledge about vaccines, address misinformation, communicate vaccine allocation decisions

Figure 1: Summary of preliminary National Advisory Committee on Immunization (NACl) recommendations on key populations for early coronavirus disease 2019 (COVID-19) immunization. Note: *Order does not indicate priority. †Based on the systematic assessment of ethics, equity, feasibility and acceptability using an evidence-informed framework. ${ }^{27}$ 
full guideline) as well as a public health ethics framework designed to guide Canada's response to the COVID-19 pandemic. ${ }^{29}$ In consultation with the Public Health Agency of Canada's (PHAC's) Public Health Ethics Consultative Group, NACI conducted an in-depth ethics analysis to weigh ethical considerations and assess options for prioritization of COVID-19 immunization in the face of an uncertain pandemic vaccine supply. Examples of NACl's integration of core ethical dimensions for public health are summarized below.

\section{Respect for persons and communities}

To respect the right to exercise informed choice, in its full guideline, $\mathrm{NACl}$ has reviewed and summarized the evidence to date for stakeholders, and recommends the transparent dissemination of information on SARS-CoV-2 vaccines and vaccine allocation decisions as a principle to guide decision-making on a COVID-19 immunization program. $\mathrm{NACl}$ considered the values and preferences of individuals and communities through an expert stakeholder survey, public opinion research and extensive consultations..$^{12-15,21}$

Table 1: Risk factors identified through a rapid review that have large or important (++), or very large or very important (+++) associations with severe COVID-19 outcomes, and the corresponding level of confidence in the association*

\begin{tabular}{|c|c|c|}
\hline Risk factort & Outcome of interest & $\begin{array}{r}\text { Magnitude of } \\
\text { (confidence in ass }\end{array}$ \\
\hline \multicolumn{3}{|l|}{ Age, yr } \\
\hline \multirow[t]{2}{*}{$>80$ v. $\leq 45$} & Hospital admission & $+++($ low $)$ \\
\hline & Death & +++ (low) \\
\hline \multirow[t]{2}{*}{$>70$ v. $\leq 45$} & Hospital admission & +++ (moderate) \\
\hline & Death & +++ (moderate) \\
\hline \multirow[t]{2}{*}{$>60 v . \leq 45$} & Hospital admission & $++/+++$ (moderate/low) \\
\hline & Death & ++/+++ (moderate/low) \\
\hline \multirow[t]{2}{*}{$50-64$ v. $\leq 45$} & Hospital admission & ++ (moderate) \\
\hline & Death & ++ (moderate) \\
\hline \multirow[t]{2}{*}{$45-54$ v. $\leq 45$} & Hospital admission & ++ (moderate) \\
\hline & Death & ++ (low) \\
\hline \multicolumn{3}{|l|}{ Pre-existing conditions } \\
\hline Obesity (BMI $\geq 40$ ) & Hospital admission & ++ (low) \\
\hline Heart failure & Hospital admission & ++ (low) \\
\hline Diabetes mellitus & Hospital admission & ++ (low) \\
\hline Liver disease & Death & ++ (low) \\
\hline Chronic kidney disease & Hospital admission & ++ (low) \\
\hline Alzheimer disease or dementia & Hospital admission & ++ (low) \\
\hline \multicolumn{3}{|l|}{ Sex } \\
\hline Male v. female & Hospital admission & ++ (moderate) \\
\hline \multicolumn{3}{|l|}{ Race or ethnicity } \\
\hline Black v. non-Hispanic White & Hospital admission & ++ (low) \\
\hline Asian (Bangladeshi) v. British White & Death & ++ (low) \\
\hline \multicolumn{3}{|l|}{ Place of residence } \\
\hline Homeless v. has a home & Hospital admission & ++ (low) \\
\hline \multicolumn{3}{|l|}{ Socioeconomic status } \\
\hline Income $\leq 25$ th $v .>50$ th or 75 th percentile & Hospital admission & ++ (low) \\
\hline
\end{tabular}

Note: $\mathrm{BMI}=$ body mass index, COVID-19 = coronavirus disease 2019, $\mathrm{NACl}$ = National Advisory Committee on Immunization.

*This table summarizes the results of a rapid review of risk factors for severe outcomes in Organisation for Economic Co-operation and Development member countries, conducted by the Alberta Research Centre for Health Evidence. ${ }^{19} \mathrm{~A}$ total of 34 published studies were included in this review. Generalization of findings from other countries to Canada should be made with caution, as high-risk groups may differ by population. Furthermore, because of differences in methodology, the list of important risk factors identified in this rapid review may differ from other sources. Updated evidence syntheses will inform future NACI decisions.

†The order of these risk factors is based on evidence appraisal and does not indicate order of priority.

$\ddagger$ Magnitude of associations are shown as large or important (++; odds ratio or risk ratio $\geq 2.00$ ), or very large or very important association (+++; odds ratio or risk ratio $\geq 5.00$ ).

$\S A$ formal assessment of the quality or confidence of the evidence was not performed but the process of assessing the quality or confidence of the evidence was based on the Grading of Recommendations, Assessment, Development and Evaluations approach (www.gradeworkinggroup.org/). Confidence in the magnitude of the associations was determined by considering primarily study limitations (risk of bias), consistency in findings across studies and precision (sample size). Low confidence indicates that there may be an association and moderate confidence means that the evidence indicates that there probably is an association. 
Table 2 (part 1 of 2): Summary of evidence for recommendations on key populations for early CoVID-19 immunization

Recommended key populations for early COVID-19 immunization*

Those at high risk of severe illness and death from COVID-19:

- Advanced age

- Other high-risk conditions (to be defined as the evidence base evolves)

Those most likely to transmit COVID-19 to those at high risk of severe illness and death from COVID-19 and workers essential to maintaining the COVID-19 response

- Health care workers, personal care workers and caregivers providing care in long-term care facilities or other congregate care facilities for seniors

- Other workers most essential in managing the COVID-19 response or providing front-line care for patients with COVID-19

- Household contacts of those at high risk of severe illness and death from COVID-19
Summary of best available evidence and rationale for the consensus recommendation

- There are large or important independent associations of severe COVID-19 outcomes with increasing age and for certain high-risk health conditions. ${ }^{19}$ There is moderate certainty of evidence for a very large or very important association of hospital admission and death, particularly in those older than 70 years (v. age $\leq 45 \mathrm{yr}$ ) and low certainty of evidence for a large or important association of hospital admission or death with certain high-risk conditions (Table 1 ). ${ }^{19}$ Studies treating age on a continuum or across small increments consistently found that risks for hospital admission and death increase with increasing age (e.g., about $2 \%-6 \%$ and $5 \%-10 \%$ relative increase in risk per year, respectively)..$^{19}$

- Current surveillance data in Canada have shown that hospital admission, ICU admission and death rates from COVID-19 increase with age, and that people with certain underlying health conditions are at highest risk of developing more severe illness from COVID-19. ${ }^{20}$

- Expert stakeholder groups and patient and community advocates, ${ }^{21}$ as well as the Canadian public, ${ }^{13}$ rank the relative importance of an immunization strategy to protect those who are most vulnerable to severe illness and death from COVID-19 as first in the context of limited vaccine supply.

- Most Canadians identified those with underlying medical conditions (57\%) and older people (53\%) as groups who should get a SARS-CoV-2 vaccine first, if supplies are limited. ${ }^{12}$ When provided with a list of specific groups to be prioritized to receive the vaccine, $19 \%$ ranked those with high-risk medical conditions and $12 \%$ ranked older adults as the top priority. ${ }^{13}$

- Older Canadians are significantly more willing than younger Canadians to get an effective recommended SARS-CoV-2 vaccine, ${ }^{12,13,15}$ and those 35 years and older with a "serious long-term illness" are somewhat more willing to get an effective recommended SARSCoV-2 vaccine..$^{12,13}$

- In Canada, long-term care facilities have experienced a large number of outbreaks associated with a high number of fatalities. ${ }^{20}$

- Immunizing health care, personal care and other workers providing front-line care directly protects them from acquiring SARS-CoV-2 infection and could indirectly protect their patients and health care capacity.

- Although front-line health care workers and other workers functioning in a health care capacity (e.g., providing medical first response) have differential exposure to SARS-CoV-2 with potential transmission to high-risk individuals, they may have more access to and training in the use of PPE and other infection prevention and control measures, and so exposure risk could be substantially reduced compared with other groups. Protection against infection with SARS-CoV-2 has been demonstrated in health care workers with the use of PPE. ${ }^{22,23}$

- Immunizing health care workers and other workers functioning in a health care capacity minimizes the disproportionate burden of those taking on additional risks to protect the public.

- Absenteeism because of illness or perceived risk of illness from COVID-19 among health care workers and other workers most essential in managing the COVID-19 response (e.g., outbreak management, laboratory testing, immunization) may compromise health care capacity and the management of the COVID-19 response.

- Expert stakeholder groups and patient and community advocates, ${ }^{21}$ as well as the Canadian public, ${ }^{13}$ rank the relative importance of an immunization strategy to protect health care capacity as second priority, and an immunization strategy to minimize transmission of COVID-19 as third in the context of limited vaccine supply.

- $22 \%$ of Canadians identify "health care workers" as a key population for priority immunization in the case of SARS-CoV-2 vaccine shortage. ${ }^{12}$ When provided with a list of specific groups to be prioritized to receive the vaccine, "health care workers" was the group most commonly ranked first by most Canadian respondents (40\%). ${ }^{13}$

- Immunizing those able to transmit SARS-CoV-2 to those at high risk of severe illness and death could indirectly protect those at high risk (if the vaccine is effective in interrupting transmission), which could be particularly important if vaccine characteristics are not favourable in high-risk populations. 
Recommended key populations for early COVID-19 immunization*
Summary of best available evidence and rationale for the consensus recommendation
Those contributing to the maintenance of other essential services for the functioning of society (to be defined, prioritized and informed by ongoing federal, provincial and territorial discussions)
- Certain individuals who cannot work virtually may have differential exposure to SARS-CoV-2.

- Designations of essential services in the context of the COVID-19 pandemic vary across jurisdictions in Canada. Guidance on essential services and functions during the COVID-19 pandemic, including lists published by provinces and territories, is available. ${ }^{24}$

- Provinces and territories have expressed a desire for a harmonized approach to vaccine prioritization for essential services. The appropriate federal, provincial and territorial health tables will be consulted in discussions on prioritization for the purposes of immunization.

- Immunizing this population minimizes the disproportionate burden of those taking on additional risks to maintain services essential for the functioning of society.

- Absenteeism because of illness or perceived risk of illness from COVID-19 among some workers who cannot work virtually may compromise essential services.

- Expert stakeholder groups and patient and community advocates ${ }^{21}$ as well as the Canadian public, ${ }^{13}$ rank the relative importance of an immunization strategy to protect critical infrastructure as fourth in the context of limited vaccine supply.

- $18 \%$ of Canadians identify "front-line or essential workers" as a key population for priority immunization in the case of SARS-CoV-2 vaccine shortage. ${ }^{12}$

Those whose living or working conditions put them at elevated risk of infection and where infection could have disproportionate consequences, including Indigenous communities (to be defined based on COVID-19 epidemiology and evidence from previous pandemics)
- In Canada, a high number of COVID-19 outbreaks or clusters in institutions (e.g. correctional facilities), work settings (e.g., agricultural or meat production or packing facilities) and congregate-living settings (e.g., shelters, migrant workers) have occurred. ${ }^{20}$

- The risk of transmission is high in these settings, where physical distancing and other infection prevention and control measures are challenging, and individuals may not be able to exercise sufficient personal actions to adequately protect themselves from infection. This increased risk may expand to other settings as they reopen.

- Remote or isolated populations or those in some congregate-living populations may not have ready access to sufficient health care infrastructure. Therefore, their risk for death and societal disruption is proportionally greater, as the response to any illness within the community might be suboptimal.

- Indigenous communities have been disproportionately affected by past pandemics (e.g., the $2009 \mathrm{H} 1 \mathrm{~N} 1$ influenza pandemic) and require special consideration of issues related to equity, feasibility and acceptability.

Note: COVID-19 = coronavirus disease 2019, PPE = personal protective equipment, SARS-CoV-2 = severe acute respiratory syndrome coronavirus 2.

${ }^{*}$ Order does not indicate priority.

\section{Beneficence and nonmaleficence}

$\mathrm{NACl}$ considered evidence for minimizing the risk of harm and maximizing benefits for all potential key populations in its deliberations. As information about vaccine characteristics (including safety and efficacy in different populations) becomes available, the principles of proportionality (measures should be proportionate to the level of risk and benefits gained), effectiveness (reasonable likelihood that the action will achieve the goals and will be feasible) and precaution (prudent action should be taken in the face of scientific uncertainty) will be applied. In the absence of direct data in populations at high risk of severe illness and death from COVID-19 (e.g., increasing age or underlying medical conditions), $\mathrm{NACl}$ will weigh these principles in an indepth ethics analysis, along with other key considerations, before making specific recommendations. Immunization strategies aimed at protecting health care capacity and other services essential for the functioning of society uphold the principle of reciprocity, as they aim to minimize the disproportionate burden faced by those taking on additional risks to protect the public.
The public also benefits from the ongoing work of those who provide these services and could potentially benefit from reduced transmission from front-line workers.

\section{Justice}

Treating people and groups with equal concern and respect entails setting and applying prioritization criteria fairly, considering the needs of those most at risk of exposure or of severe disease, and weighing risks of furthering inequities, stigmatization and discrimination. $\mathrm{NACl}$ reviewed special considerations for those most at risk, as well as other factors such as systemic marginalization of groups with differential disease severity and differential access to health care (see Equity Matrix, Appendix D in the full guideline). In addition to its examination of equity issues, $\mathrm{NACl}$ considered distributive justice (the fair deployment of resources) with its application of the Feasibility Matrix (Appendix $\mathrm{E}$ in the full guideline). As a result, $\mathrm{NACl}$ has recommended that equity and feasibility be integrated as principles to guide decision-making on a COVID-19 immunization program. 
Table 3: Summary of evidence for recommendations on principles to guide public health program-level decision-making on a COVID-19 immunization program

\section{Summary of best available evidence and rationale for the consensus recommendation}

Recommended guiding principle for decision-making*

Efforts should be made to increase access to immunization services to reduce health inequities without further stigmatization or discrimination, and to engage systematically marginalized and racialized populations in immunization program planning (see NACl's Equity Matrix, Appendix D in the full guideline).
- Health inequities exist in part because of differential access to health care, as well as differential exposure, susceptibility and severity of infectious diseases (see Equity Matrix, Appendix D in the full guideline). Interventions to reduce these inequities rather than potentiate them with further stigmatization or discrimination should be implemented as part of any immunization program.

- As with any immunization program, efforts should be made to ensure consideration of the needs of diverse population groups, based on health status, ethnicity or culture, ability, and other socioeconomic and demographic factors that may place individuals in vulnerable circumstances (e.g., occupational, social, economic or biological vulnerabilities). These efforts should include integrating the values and preferences of these populations in vaccine program planning and building capacity to ensure access and convenience of immunization services.

- There is evidence of important or large independent associations of severe COVID-19 with race or ethnicity (low certainty of evidence of hospital admission or death), low socioeconomic status (low certainty of evidence for hospital admission), homelessness (low certainty of evidence for hospital admission), and male sex (moderate certainty of evidence for hospital admission). ${ }^{19}$

- Outbreaks involving large numbers of reported cases have occurred in rural and remote communities in Canada. ${ }^{20}$

- Outbreaks involving large numbers of reported cases have occurred in agricultural work settings, including those with congregate living for migrant workers. ${ }^{20}$

- Visible minorities and Indigenous Canadians appear to be less willing than nonvisible minorities to get an effective recommended SARS-CoV-2 vaccine. ${ }^{12}$ Reasons for vaccine hesitancy are multifactorial.

- Although significant differences in willingness to get vaccinated with a SARS-CoV-2 vaccine have not been observed by sex or socioeconomic status, ${ }^{12,13}$ immunization coverage has tended to be lower among men and those in lower socioeconomic groups for vaccine-preventable diseases, where national data are available. ${ }^{25}$

- Examples of interventions to engage communities and address barriers to accessing vaccine, as summarized in the Equity Matrix (Appendix D in the full guideline), could help reduce inequities.

- The principle of equity urges consideration of health and economic disparities to ensure a fair distribution of resources.

- Stakeholder reviews of feasibility identified multiple challenges requiring advanced planning and complex combinations of program administration through a variety of vaccine delivery models across Canada. for the implementation of a COVID-19 immunization program, including rapid monitoring of safety, effectiveness and coverage of vaccine(s) in different key populations, as well as effective and efficient immunization of populations in remote and isolated communities (see Feasibility Matrix, Appendix E in the full guideline).

Efforts should be made to improve knowledge about the benefits of vaccines in general and of COVID-19 vaccine(s) specifically, once available, to address misinformation about immunization, and to communicate transparently about COVID-19 vaccine allocation decisions (see Acceptability Matrix, Appendix F in the full guideline).
- Planning is required to address issues specific to a potential COVID-19 immunization program (e.g., storage and dissemination of new vaccine technologies in different vaccine delivery venues; human resources for administration of vaccine, communication, training, data entry, screening for COVID-19, security of supplies, operational planning, etc.), and integration with or enhancement of existing programs (e.g., registries, surveillance, adverse event after immunization reporting).

- Rapid monitoring of safety, effectiveness and coverage of the vaccine(s) in potentially different key populations will be critical.

- The feasibility of sequential immunization of different key populations in remote and isolated communities is challenging. In these settings, deploying vaccine to entire communities may be more effective and efficient than sequential immunization of different key populations.

- Willingness to get a safe, effective SARS-CoV-2 vaccine has decreased over time in Canada (from $71 \%$ in April to $61 \%$ in August 2020). ${ }^{9}$ The most reported reasons for unwillingness to get a SARS-CoV-2 vaccine across various Canadian surveys are concerns about safety and a lack of confidence and trust in a new SARS-CoV-2 vaccine. ${ }^{12-15}$

- Deemed 1 of the top 10 major global health threats by WHO in 2019, vaccine hesitancy could limit the success of a COVID-19 immunization program. Key reasons for vaccine hesitancy include complacency, inconvenience in accessing vaccines and lack of confidence. ${ }^{16}$

- Efforts should be made to reduce complacency, improve convenient access to vaccines, and improve confidence in and awareness of immunization in the public, key populations for early COVID-19 immunization and health care providers. Transparent, clear communication about vaccine trials, pharmacovigilance ${ }^{26}$ and vaccine allocation decisions is important to maintain trust and confidence, and improve access to vaccines for key populations.

- In general, receiving a recommendation from, or being in contact with a health care provider, is linked to increased vaccine acceptability,${ }^{17}$ and a notable factor for health care providers to recommend a vaccine is a recommendation by an expert committee. ${ }^{18}$

Note: COVID-19 = coronavirus disease $2019, \mathrm{NACl}=$ National Advisory Committee on Immunization, SARS-CoV-2 = severe acute respiratory syndrome coronavirus 2 , WHO = World Health Organization.

*Order does not indicate priority. 
Table 4: Definitions adopted for the application of the Ethics, Equity, Feasibility, Acceptability Framework ${ }^{27}$

Factor

Ethics

Equity

Feasibility

Acceptability
Definition

A systematic process to clarify, prioritize and justify possible courses of action based on ethical principles, and involves the application of relevant principles and values to public health decision-making.

The absence of avoidable, unfair or remediable differences among groups of people, whether those groups are defined socially, economically, demographically or geographically or by other means of stratification. "Health equity" or "equity in health" implies that ideally, everyone should have a fair opportunity to attain their full health potential and that no one should be disadvantaged from achieving this potential. ${ }^{28}$

The potential for a program to be successfully implemented in the local setting with available resources.

A marker of desirability or demand for a given product or program, including intention and behaviours toward vaccination.

\section{Trust}

Reliability and integrity of guidance must be maintained for trust in this and other immunization programs. Expedited regulatory reviews of SARS-CoV-2 vaccines and evolving evidence about the disease may have an effect on the trust of the public in this immunization program and their perception of risks associated with SARS-CoV-2 vaccines. NACI followed its established methodology, standard operating procedures and conflict of interest guidelines ${ }^{30}$ in the context of this urgent review to ensure a robust analysis of evidence and to maintain stakeholder trust. This guidance is based on the best current evidence available for all groups at risk of COVID-19, with transparency about knowns and unknowns, as well as certainty of evidence. $\mathrm{NACl}$ will monitor the evidence with revision of guidance as necessary.

\section{Equity}

The inequitable allocation of scarce resources could exacerbate existing inequities that have been magnified in the COVID19 pandemic, and slow its resolution. ${ }^{31}$ Health inequities exist in part because of differential access to health care, as well as differential exposure, susceptibility and severity of infectious diseases (Table 1). Interventions to reduce these inequities rather than perpetuate them with further stigmatization or discrimination should be implemented as part of any immunization program.

$\mathrm{NACl}$ reviewed the epidemiology of COVID-19 in Canada ${ }^{20}$ and the results of a rapid review of risk factors for severe COVID-1919 to identify distinct inequities associated with COVID-19, potential reasons for and intersections between the full spectrum of inequities where evidence does or does not currently exist, and suggested interventions to reduce inequities and improve access to a vaccine when it becomes available. Diverse interventions that are inclusive and respectful of a pluralistic society, including racialized and systemically marginalized populations, are needed. The results of NACl's analysis are summarized in the Equity Matrix (Appendix D in the full guideline) and expanded on in a separate commentary. ${ }^{31}$

Equity is a guiding principle in this and future guidance on COVID-19 immunization programs; this principle has been integrated into previous $\mathrm{NACl}$ guidance on research priorities for SARS-CoV-2 vaccines. ${ }^{32} \mathrm{NACl}$ continues to urge that individuals with potential vulnerabilities to disease related to biological, social and occupational factors be included in clinical trials as soon as possible, and that vaccine outcome results be disaggregated by potential factors contributing to inequities, such as sex, age, race or ethnicity, and health status. Trials should endeavour to power their studies to allow analyses by various sociodemographic variables.

\section{Feasibility}

$\mathrm{NACl}$ recognizes that there are challenges to the feasible implementation of a COVID-19 immunization program with respect to resources (e.g., vaccine storage and immunization supplies) as well as integration with existing programs (e.g., immunization registries, surveillance) for potentially different vaccines. To assist jurisdictions with the planning of a COVID-19 immunization program, these issues are summarized in Appendix $\mathrm{E}$ of the full guideline. Clarity of recommendations for different target groups as well as rapid monitoring of safety, effectiveness and coverage of the vaccine(s) in key populations will be critical.

The feasibility of immunizing different populations will vary with the size of the population to be immunized, vaccine characteristics in the population, access to immunization services, and so on. In some Indigenous communities, crowded multigenerational living makes segregation of at-risk groups challenging, and precarious supply chains, infrastructure and health systems are vulnerable to critical disruption. In these cases, there may be value in implementing multiple strategies concurrently and completely immunizing entire communities rather than sequentially immunizing different key populations.

\section{Acceptability}

$\mathrm{NACl}$ considered the values and preferences of a range of stakeholders, including experts, the public, and patient and community advocates.

A survey of 156 expert stakeholders on the relative importance of COVID-19 immunization strategies ${ }^{21}$ showed that they generally ranked the strategies in the following order, from most to least important: protect those who are most vulnerable to severe illness and death from COVID-19, protect health care capacity, minimize transmission of COVID-19 and protect critical infrastructure.

$\mathrm{NACl}$ also reviewed the results of a longitudinal online survey of a representative sample of about 2000 Canadians, which lever- 
aged a tool developed by the WHO to monitor knowledge, risk perceptions and behaviour related to COVID-19. ${ }^{12,13} \mathrm{NACl}$ reviewed public opinion research to understand which immunization strategies and populations Canadians would prioritize if SARS-CoV-2 vaccine supply is limited, how acceptability of a SARS-CoV-2 vaccine varies by sociodemographic factors, and what factors affect acceptability of a SARS-CoV-2 vaccine. ${ }^{12-15}$ These findings are summarized in the Acceptability Matrix (Appendix F in the full guideline). Previously conducted research to validate NACl's Acceptability Matrix before the COVID-19 pandemic was consistent with recent surveys on SARS-CoV-2 vaccines, which showed that concern about vaccine safety is the main reason among Canadians for reluctance to be vaccinated. ${ }^{17,27}$

$\mathrm{NACl}$ acknowledges that survey data on intent to receive a vaccine can fluctuate, especially in the context of uncertainty about possible SARS-CoV-2 vaccines. Furthermore, online surveys may not capture the values and preferences of all populations. NACl will continue to monitor studies assessing acceptability of SARS-CoV-2 and other vaccines among Canadians and encourages use of other research methodologies to understand the values and preferences of marginalized populations.

\section{Methods}

\section{Guideline panel composition}

$\mathrm{NACl}$ is an external advisory body to $\mathrm{PHAC}^{33}$ that provides advice on the use of vaccines and passive immunizing agents in humans in Canada (www.canada.ca/naci).

The committee is composed of 14 voting members, who are recruited through an open nomination process and appointed based on, but not limited to, their expertise, knowledge and experience in immunization, public health, vaccine-preventable diseases, pediatric or adult infectious diseases, allergy or immunology, and other health-related fields such as pharmacy and nursing, pharmacoeconomics, epidemiology and infectious disease modelling, as well as their previous experience on vaccine advisory committees.

Representatives of health professional associations and national committees participate in $\mathrm{NACl}$ activities as liaison members. Representatives from federal government departments participate as ex-officio members. Liaison and ex-officio members do not have voting status.

NACl's work on SARS-CoV-2 vaccines is led by its High Consequence Infectious Disease Working Group (HCID WG), which proposes draft recommendations for NACl's deliberation and approval. The HCID WG comprises 12 members, including $5 \mathrm{NACl}$ members (including S.D., C.Q.), 4 external experts, 2 liaison members (from the Committee to Advise on Tropical Medicine and Travel and the Centers for Disease Control and Prevention's Advisory Committee on Immunization Practices) and 1 ex-officio member (from Health Canada).

The committee reports to the vice-president of the Infectious Disease Prevention and Control Branch of PHAC. PHAC provides scientific, project management and logistical support to $\mathrm{NACl}$ through the NACI Secretariat. All NACI and HCID WG members are volunteers and not remunerated for their services.
A complete list of NACI members, HCID WG members and $\mathrm{NACl}$ Secretariat staff from PHAC who participated in the development of this guideline can be found in the Contributors' section of this article.

\section{Key question}

As $\mathrm{NACl}$ is an external advisory body to PHAC, PHAC formulated the key question for $\mathrm{NACl}$ based on policy needs. The key question addressed by this guideline is, "Assuming constrained early supply for SARS-CoV-2 vaccine(s) in Canada, which populations should be prioritized for early doses of vaccine?"

\section{Guideline development process}

Details of NACl's recommendation development process can be found elsewhere. ${ }^{27,30}$ In brief, the stages for the development of $\mathrm{NACl}$ recommendations are evidence retrieval (including stakeholder consultation), evidence synthesis and translation of the summarized evidence into recommendations. The relevant $\mathrm{NACl}$ working group (HCID WG in this case) is responsible for establishing the scope of and requirements for the evidence review. The $\mathrm{NACl}$ Working Group reviews and discusses the summarized evidence and then proposes recommendation options for NACl's consideration.

After full committee review and discussion of the evidence and recommendation options, $\mathrm{NACl}$ votes on the options. A quorum of at least two-thirds of members is required to authenticate a vote. The final $\mathrm{NACl}$ guideline, incorporating committee discussion and vote, is circulated by email for approval. After this approval and final review by the $\mathrm{NACl}$ Chair, the guideline is sent to the vice-president of the Infectious Disease Prevention and Control Branch to approve public release.

\section{Evidence retrieval}

To develop this guideline over the summer of $2020, \mathrm{NACl}$ reviewed available epidemiologic summaries from national analyses of federal, provincial and territorial surveillance data reported to PHAC (https://health-infobase.canada.ca/covid-19/ epidemiological-summary-covid-19-cases.html); summaries of the SARS-CoV-2 vaccine product landscape from clinical trial registry data (www.who.int/publications/m/item/draft-landscape -of-covid-19-candidate-vaccines); and the Vaccine Annex of the Canadian Pandemic Influenza Preparedness: Planning Guidance for the Health Sector. ${ }^{34}$

In addition, the NACI Secretariat (S.I., L.Z., M.T.) conducted an environmental scan of international guidance on prioritization of key populations for initial COVID-19 immunization (summarized in Table 5). The NACI Secretariat commissioned the Alberta Research Centre for Health Evidence to conduct a rapid review of risk factors for severe COVID-19 (literature search carried out on June 15, 2020). Full methodological details and results can be found in the preprint (www.medrxiv.org/content/10.1101/2020.08 $.27 .20183434 \mathrm{v} 1) .{ }^{19}$

The NACI Secretariat applied NACl's Ethics, Equity, Feasibility and Acceptability (EEFA) Framework, ${ }^{27}$ using the published algorithm (Appendix A in the full guideline). The secretariat populated the evidence-informed tools that accompany the frame- 
Table 5: Summary of interim guidance on COVID-19 immunization from other national immunization technical advisory groups

National immunization technical

advisory group

Summary of recommendations on priority groups

Joint Committee on Vaccination and Immunization, United Kingdom³5
The updated interim advice on priority groups for COVID-19 vaccination uses a combination of clinical risk stratification and an age-based approach with the provisional ranking of prioritization for persons at risk, as follows:

- Older adult residents in a care home and care home workers

- All those aged 80 years and older, and health and social care workers

- All those aged 75 years and older

- All those aged 70 years and older

- All those aged 65 years and older

- High-risk adults younger than 65 years

- Moderate-risk adults younger than 65 years

- All those aged 60 years and older

- All those aged 55 years and older

- All those aged 50 years and older

- Rest of the population (priority to be determined)

The committee noted that the prioritization could change substantially if the first available vaccines were not considered suitable for, or effective in, older adults.

Haute Autorité de Santé, France ${ }^{36}$
The preliminary advice identified key populations for vaccine prioritization based on health risk and occupational risk, whether they were critical workers, and socioeconomic and demographic characteristics, as follows:

\section{First priority:}

1. Population at risk of occupational exposure (health personnel or those working with vulnerable groups; as well as front-line workers, people working in confined areas and workers with confinedaccommodation arrangements)

2. People at higher risk because of their age or health conditions, both in metropolitan France and overseas departments and regions of France

3. People living in precarious situations

\section{Secondary priority:}

1. Populations of the overseas departments and regions of France in the event of a shortage of resuscitation beds (and who do not belong to the groups that have already been prioritized)

2. People living in closed establishments at increased risk of transmission (e.g., prisons, establishments for people with disabilities, psychiatric hospitals)

3. Personnel with strategic jobs (e.g., police officers, firefighters, active military personnel)

Note: COVID-19 = coronavirus disease 2019, JCVI = Joint Committee on Vaccination and Immunization.

*An older version of the JCVI interim advice on priority groups for COVID-19 vaccination (June 18, 2020) was reviewed by the National Advisory Committee on Immunization. In the now withdrawn report, JCVI advised priority recommendation of front-line health and social care workers and those at increased risk of serious disease and death from COVID-19 infection, stratified according to age and risk factors.

work to identify distinct issues that could affect decision-making, consulting relevant groups and data as needed. The secretariat presented the tools to the HCID WG and $\mathrm{NACl}$; the tools were also included as appendices in the full guideline (B, C, D, E and F).

$\mathrm{NACl}$ and the HCID WG deliberated the key considerations during decision-making; distinct issues that could have an effect on recommendations or implementation of the recommendations, as well as suggested interventions to address these issues, are summarized in the full guideline.

\section{Stakeholder consultation}

The NACI Secretariat conducted a national survey of expert stakeholders (including clinical and public health experts, members of provincial and territorial committees, representatives from national Indigenous groups, patient and community advocates, executives of Canadian health professional associations, and representatives of federal government departments) between July 22 and Aug. 14, 2020, to establish a comprehensive perspective on the relative importance of pandemic immunization strategies under 4 different pandemic scenarios at the time of initial SARSCoV-2 vaccine availability. Respondents were not required to declare potential conflicts of interest, representing a major limitation of this survey. A follow-up survey to the public is planned to validate the results of this expert stakeholder survey. ${ }^{37}$ Full methodological details and results of the expert stakeholder survey can be found in the preprint (www.medrxiv.org/content/10.1101/ 2020.09.16.20196295v1). ${ }^{21}$

$\mathrm{NACl}$ consulted the following stakeholder groups during the development of this guideline: PHAC's Public Health Ethics Consultative Group, PHAC's Sex and Gender Based Analysis+ net- 
work, Indigenous Services Canada and other NACI liaison and exofficio organizations.

$\mathrm{NACl}$ also consulted the following federal, provincial and territorial committees: Canadian Immunization Committee, the Pan-Canadian Public Health Network's Special Advisory Committee on COVID-19, and the Special Advisory Committee's Technical Advisory Committee. The Canadian Immunization Committee, Indigenous Services Canada and PHAC's vaccine supply manager reviewed the Feasibility Matrix of NACl's EEFA Framework.

Additional consultations with groups representing vulnerable populations are planned to inform the development of further guidelines on key populations for early COVID-19 immunization.

\section{Evidence synthesis and development of recommendations}

The NACl Secretariat synthesized the results of the evidence reviews for consideration by the $\mathrm{HCID} W \mathrm{WG}$ and $\mathrm{NACl}$ in formulating the key populations for early COVID-19 immunization.

The HCID WG reviewed and discussed available evidence and relevant considerations on June 26 and July 24, 2020. NACI reviewed and deliberated on the evidence and recommendation options on Aug. 13, 2020, and again on Aug. 20, 2020, at a joint meeting with the HCID WG. These meetings were held virtually. At the meeting on Aug. 20, 2020, NACl members voted unanimously in favour of the proposed recommendations.

The secretariat drafted the guideline with input from the $\mathrm{NACl}$ Chair (C.Q.) and Vice-Chair (S.D.). The HCID WG reviewed the draft guideline in July 2020. The following federal, provincial and territorial committees reviewed the draft guideline in August 2020: the Canadian Immunization Committee, the Pan-Canadian Public Health Network's Special Advisory Committee on COVID-19, and the Special Advisory Committee's Technical Advisory Committee. These groups provided input to $\mathrm{NACl}$ for consideration. $\mathrm{NACl}$ approved the guideline through an email process on Sept. 16, 2020.

\section{Management of competing interests}

PHAC has an established process for assessing and managing competing interests in guidance development. This process is informed by the Guidelines International Network principles for disclosing and managing competing interests. ${ }^{38}$ The $\mathrm{NACl}$ Chair, Vice-Chair, all $\mathrm{NACl}$ voting and liaison members, and all HCID WG members were required to disclose financial and nonfinancial competing interests, using a standardized disclosure of interest form, and to declare any new interests at the beginning of meetings. PHAC assessed declared interests via the NACI Secretariat, using established evaluation criteria informed by the Guidelines International Network principles.

Members did not declare any interests that represented direct or indirect financial or nonfinancial conflicts of interest for this guideline. Specifically, there were no interests relating to SARS-CoV-2 vaccine candidates with manufacturers with advance purchase agreements with the Government of Canada at the time of development. Had any conflict of interest been identified, a management strategy would have been put in place whereby affected members would abstain from voting on, discussing or observing recommendation development related to the conflict, depending on the extent of the conflict. No such restrictions were necessary during this guideline development.

\section{Implementation}

The recommendations in this guideline are preliminary as they were developed in the context of many unknowns. Therefore, $\mathrm{NACl}$ did not prioritize the key populations for early COVID-19 immunization. Specific guidance and $\mathrm{NACl}$ prioritization of key populations for early COVID-19 immunization will be feasible as more information about SARS-CoV-2 vaccine(s) authorized for use in Canada become(s) available.

\section{Other guidelines}

The key populations that NACI has identified for early COVID-19 immunization have been similarly identified by other national immunization technical advisory groups in their interim guidance $^{35,36}$ (Table 5), and academic groups in their vaccine prioritization frameworks. ${ }^{39,40}$

The United Kingdom's Joint Committee on Vaccination and Immunization proposed a combined risk- and age-based approach with the sequencing of key populations for early SARSCoV-2 vaccination, ${ }^{35}$ while France's Haute Autorité de Santé proposed a risk-based approach to prioritization. ${ }^{36}$

PHAC is in regular contact with secretariats of established national immunization technical advisory groups in order to align the evidence base for guidance where possible. Furthermore, the United States Advisory Committee on Immunization Practices (ACIP) holds a liaison position to $\mathrm{NACl}$, and $\mathrm{NACl}$ holds a liaison position to ACIP.

\section{Gaps in knowledge}

Research to address outstanding questions (outlined in the full guideline) in the following areas is encouraged: SARS-CoV-2 vaccine characteristics in diverse population groups (including safety, efficacy, duration of protection, effectiveness in preventing severe illness and death, and interrupting transmission); correlates of protection against SARS-CoV-2; epidemiologic profile of COVID-19; effectiveness and safety of other preventive or therapeutic interventions for COVID-19; acceptability of SARS-CoV-2 vaccines; and inequities in COVID-19 and interventions to reduce inequities and improve acceptability related to COVID-19 immunization.

\section{Limitations}

This guidance is based on the evidence available at the time of $\mathrm{NACl}$ deliberations, along with expert and stakeholder opinion. Evidence on COVID-19 and SARS-CoV-2 vaccines is rapidly evolving, and values and preferences of stakeholders may change. Because of the urgent need for this guideline, consultations with groups representing vulnerable populations were limited. More extensive consultations are planned to inform further guidance. 
Key populations may change as the evidence base for COVID19 and SARS-CoV-2 vaccine characteristics, as well as information on vaccine supply, evolve. Prioritization is not yet possible without data to support whether direct protection of key populations (e.g., advanced age) will be possible or whether indirect protection will be a more effective strategy. $\mathrm{NACl}$ will provide further guidance when additional information on vaccine-specific characteristics in different populations becomes available.

\section{Conclusion}

$\mathrm{NACl}$ has developed preliminary recommendations on key populations for early COVID-19 immunization to inform public health program planning for the efficient, effective and equitable allocation of SARS-CoV-2 vaccine(s). NACI's recommendations have been and will continue to be based on a population-based riskbenefit analysis, taking into consideration risk of exposure, risk of transmission to others and risk of severe illness and death.

Key populations are currently identified as populations at increased risk of severe disease, those at risk of transmitting to high-risk individuals, essential workers, and those in settings where risk is elevated. From the data currently available, it is clear that health equity will be central to dictating prioritization once results of clinical trials become available.

Existing inequities magnified by this pandemic may be exacerbated with the inequitable allocation of vaccines. Efforts should be made to increase access to immunization services and engage racialized and systemically marginalized populations in immunization program planning. The integration of equity, feasibility and acceptability considerations across all populations is critical for decisions regarding a COVID-19 immunization program.

\section{References}

1. Coronavirus disease 2019 (COVID-19): situation report - 51. Geneva: World Health Organization; 2020 Mar. 11. Available: www.who.int/docs/default-source/ coronaviruse/situation-reports/20200311-sitrep-51-covid-19.pdf?sfvrsn=1ba62e57 _10 (accessed 2020 July 11).

2. Coronavirus disease (COVID-19): outbreak update. Ottawa: Government of Canada; modified 2020 Sept. 13. Available: www.canada.ca/en/public-health/services/ diseases/2019-novel-coronavirus-infection.html (accessed 2020 Oct. 15).

3. Federal/provincial/territorial public health response plan for ongoing management of COVID-19. Ottawa: Government of Canada; modified 2020 Aug. 25. Available: www.canada.ca/en/public-health/services/diseases/2019-novel -coronavirus-infection/guidance-documents/federal-provincial-territorial-public -health-response-plan-ongoing-management-covid-19.html (accessed 2020 Aug. 26).

4. Coronavirus disease (COVID-19): for health professionals. Ottawa: Government of Canada; modified 2020 Oct. 10. Available: www.canada.ca/en/public-health/ services/diseases/2019-novel-coronavirus-infection/health-professionals.html (accessed 2020 Sept. 24).

5. Benefits of immunization: Canadian immunization guide. Ottawa: Government of Canada; 2016. Available: www.canada.ca/en/public-health/services/publications /healthy-living/canadian-immunization-guide-part-1-key-immunization-information /page-3-benefits-immunization.html (accessed 2020 June 21).

6. Draft landscape of COVID-19 candidate vaccines. Geneva: World Health Organization; 2020 Oct. 15. Available: www.who.int/publications/m/item/draft-landscape -of-covid-19-candidate-vaccines (accessed 2020 Oct. 15).

7. Drugs and vaccines for COVID-19. Ottawa: Government of Canada; modified 2020 Oct. 12. Available: www.canada.ca/en/health-canada/services/drugs-health -products/covid19-industry/drugs-vaccines-treatments/about.html (accessed 2020 Oct. 15).
8. Moderna TX. Inc. A study to evaluate efficacy, safety, and immunogenicity of mRNA-1273 vaccine in adults aged 18 years and older to prevent COVID-19. ClinicalTrials.gov: NCT04470427; 2020. Available: https://clinicaltrials.gov/ct2/ show/NCT04470427 (accessed 2020 Oct. 14).

9. BioNTech SE. Study to describe the safety, tolerability, immunogenicity, and efficacy of RNA vaccine candidates against COVID-19 in healthy individuals. ClinicalTrials.gov: NCT04368728; 2020. Available: https://clinicaltrials.gov/ct2/ show/NCT04368728 (accessed 2020 Oct. 14).

10. Janssen Vaccines \& Prevention B.V. A study of Ad26.COV2.S for the prevention of SARS-CoV-2-mediated COVID-19 in adult participants (ENSEMBLE). ClinicalTrials.gov: NCT04505722; 2020. Available: https://clinicaltrials.gov/ct2/ show/NCT04505722?term=NCT04505722 (accessed 2020 Oct. 14).

11. AstraZeneca. Phase III double-blind, placebo-controlled study of AZD1222 for the prevention of COVID-19 in adults. ClinicalTrials.gov: NCT 04516746; 2020. Available: https://clinicaltrials.gov/ct2/show/NCT04516746 (accessed 2020 Oct. 14).

12. Wave 6 results. Ottawa: Government of Canada - Impact Canada; 2020. Available: https://impact.canada.ca/en/challenges/cosmo-canada/wave6 (accessed 2020 Aug. 17).

13. Wave 7 results. Ottawa: Government of Canada - Impact Canada; 2020. Available: https://impact.canada.ca/en/challenges/cosmo-canada/wave7 (accessed 2020 Aug. 17).

14. COVID-19: three-in-five worry about side-effects of a vaccine; many plan to take a 'wait and see' approach. Angus Reid Institute; 2020. Available: http://angusreid .org/wp-content/uploads/2020/08/2020.08.03_COVID-VACCINE.pdf (accessed 2020 Aug. 17).

15. Frank K, Arim R. StatCan COVID19: data to insights for a better Canada. Group differences and reasons for vaccine hesitancy. Ottawa: Statistics Canada; 2020 Aug. 25. Available: https://www150.statcan.gc.ca/n1/pub/45-28-0001/2020001 /article/00073-eng.htm (accessed 2020 Aug. 25).

16. Ten threats to global health in 2019. Geneva: World Health Organization;2019. Available: www.who.int/news-room/spotlight/ten-threats-to-global-health-in -2019 (accessed 2020 Aug. 21).

17. Gates A, Gates M, Rahman S, et al. A systematic review of factors that influence the acceptability of vaccines among Canadians. Vaccine. In press.

18. Environics Research. Vaccine acceptability factors for the general public and health care professionals in Canada. Ottawa: Health Canada; 2020.

19. Wingert A, Pillay J, Gates M, et al. Risk factors for severe outcomes of COVID-19: a rapid review. medRxiv 2020 Sept. 1. doi: 10.1101/2020.08.27.20183434

20. Epidemiological summary of COVID-19 cases in Canada. Ottawa: Government of Canada; 2020. Available: https://health-infobase.canada.ca/covid-19 /epidemiological-summary-covid-19-cases.html (accessed 2020 Oct. 15).

21. Zhao L, Ismail SJ, Tunis MC. Ranking the relative importance of immunization strategies for novel coronavirus disease 2019 (COVID-19): a rapid survey of stakeholders. medRxiv 2020. doi: 10.1101/2020.09.16.20196295

22. Liu M, Cheng SZ, Xu KW, et al. Use of personal protective equipment against coronavirus disease 2019 by healthcare professionals in Wuhan, China: cross sectional study. BMJ 2020;369:m2195.

23. Wang W, Min YZ, Yang CM, et al. Association of personal protective equipment use with successful protection against COVID-19 infection among health care workers. medRxiv 2020 Apr. 28. doi: 10.1101/2020.04.24.20070169

24. Guidance on essential services and functions in canada during the COVID-19 Pandemic. Ottawa: Public Safety Canada; 2020. Available: www.publicsafety. gc.ca/cnt/ntnl-scrt/crtcl-nfrstrctr/esf-sfe-en.aspx (accessed 2020 Aug. 21).

25. Roy M, Sherrard L, Dubé È, et al. Determinants of non-vaccination against seasonal influenza. Health Rep 2018;29:12-22.

26. Vaccine safety and pharmacovigilance: Canadian immunization guide. Ottawa: Government of Canada; 2019. Available: www.canada.ca/en/public-health/services /publications/healthy-living/canadian-immunization-guide-part-2-vaccine-safety/ page-2-vaccine-safety.html (accessed 2020 Aug. 21).

27. Ismail SJ, Hardy K, Tunis MC, et al. A framework for the systematic consideration of ethics, equity, feasibility, and acceptability in vaccine program recommendations. Vaccine 2020;38:5861-76.

28. Health equity. Geneva: World Health Organization. Available: www.who.int/ topics/health_equity/en/ (accessed 2020 May 22).

29. Public health ethics framework: a guide for use in response to the COVID-19 pandemic in Canada. Ottawa: Government of Canada; modified 2020 June 5. Available: www.canada.ca/en/public-health/services/diseases/2019-novel -coronavirus-infection/canadas-reponse/ethics-framework-guide-use-response -covid-19-pandemic.html (accessed 2020 June 21).

30. Ismail SJ, Langley JM, Harris TM, et al. Canada's National Advisory Committee on Immunization (NACl): evidence-based decision-making on vaccines and immunization. Vaccine 2010;28:A58-63. 
31. Ismail S, Tunis M, Zhao L, et al. Navigating inequities: a roadmap out of the pandemic. SSRN 2020 Sept. 21. doi: 10.2139/ssrn.3695697

32. Research priorities for COVID-19 vaccines to support public health decisions. Ottawa: Government of Canada - National Advisory Committee on Immunization; modified 2020 July 15. Available: www.canada.ca/en/public-health/services /immunization/national-advisory-committee-on-immunization-naci/research -priorities-covid-19-vaccines.html (accessed 2020 Aug. 21).

33. External advisory bodies for the Public Health Agency of Canada. Ottawa: Government of Canada; 2016. Available: www.canada.ca/en/public-health/corporate/ mandate/about-agency/external-advisory-bodies.html (accessed 2020 Sept. 24).

34. Vaccine annex: Canadian pandemic influenza preparedness: planning guidance for the health sector. Ottawa: Public Health Agency of Canada; 2017. Available: www.canada.ca/en/public-health/services/flu-influenza/canadian -pandemic-influenza-preparedness-planning-guidance-health-sector/vaccine -annex.html (accessed 2020 May 22).

35. Joint Committee on Vaccination and Immunization. Interim advice on priority groups for COVID-19 vaccination. United Kingdom: Department of Health and Social Care; 2020 June 18. Available: www.gov.uk/government/publications/ priority-groups-for-coronavirus-covid-19-vaccination-advice-from-the-jcvi-25 -september-2020 (accessed 2020 Sept. 25).
36. Stratégie vaccinale contre la Covid-19: stratégie de déploiement des vaccins disponibles. Saint-Denis (FR): Haute Autorité de Santé; 2020 July 13. Available: www. has-sante.fr/upload/docs/application/pdf/2020-07/note_de_cadrage_strategie_ vaccinale_contre_la_covid_19.pdf (accessed 2020 Aug. 21).

37. MacDonald SE, Tunis MC, Gagneur A. Vaccination in a pandemic: the impact on routine vaccinations and future COVID-19 vaccine acceptance. Ottawa: Government of Canada; 2020. Available: https://webapps.cihr-irsc.gc.ca/decisions/p/ project_details.html?applld=430340 (accessed 2020 Sept. 24).

38. Schünemann HJ, Al-Ansary LA, Forland F, et al. Guidelines International Network: principles for disclosure of interests and management of conflicts in guidelines. Ann Intern Med 2015;163:548-53.

39. Toner E, Barnill A, Krubiner C, et al. Interim framework for COVID-19 vaccine allocation and distribution in the United States. Baltimore: Johns Hopkins Center for Health Security; 2020.

40. Committee on Equitable Allocation of Vaccine for the Novel Coronavirus. National Academies release draft framework for equitable allocation of a COVID-19 vaccine, seek public comment. Washington (D.C): National Academies of Sciences Engineering Medicine; 2020 Sept. 1. Available: www.nationalacademies.org/news /2020/09/national-academies-release-draft-framework-for-equitable-allocation -of-a-covid-19-vaccine-seek-public-comment (accessed 2020 Sept. 24).
Note: CMAJ uses the term "SARS-CoV-2" to refer to the virus and "COVID-19" to refer to the disease. In some places in this article, "COVID19 " is used instead of "SARS-CoV-2" to maintain consistency with the full National Advisory Committee on Immunization guideline (available at www.canada.ca/naci).

Competing interests: Members of the National Advisory Committee on Immunization $(\mathrm{NACl})$ are recognized experts who volunteer their time and are not remunerated for their contributions to $\mathrm{NACl}$ guidance. All authors declare that they have no known competing financial interests or personal relationships that could have, or could appear to have, influenced the work reported in this paper. Shainoor Ismail, Linlu Zhao and Matthew Tunis are employees of the Public Health Agency of Canada. Shelley Deeks is an employee of Public Health Ontario. Shelley Deeks and Caroline Quach have both received grants to conduct research on immunization issues unrelated to SARS-CoV-2 vaccines. No other competing interests were declared.

This article has been peer reviewed.

Affiliations: Centre for Immunization and Respiratory Infectious Diseases (Ismail, Zhao, Tunis), Public Health Agency of Canada, Ottawa, Ont.; Metro City Medical Clinic (Ismail), Edmonton, Alta.; Public Health Ontario (Deeks); Dalla Lana School of Public Health (Deeks), University of Toronto, Toronto, Ont.; Department of Microbiology, Infectious Diseases \& Immunology (Quach), University of Montreal; Department of Clinical Laboratory Medicine (Quach), CHU Sainte-Justine, Montréal, Que.

Contributors: Shainoor Ismail drafted the original manuscript of this summary of the full guideline. All of the named authors reviewed and revised the manuscript and gave final approval of the version to be published and agree to be accountable for all aspects of the work. The National Advisory Committee on Immunization (NACl) Chair (Caroline Quach); NACI Vice-Chair (Shelley Deeks); NACl voting members (Julie A. Bettinger, Natalie Dayneka, Philippe De Wals, Ève Dubé, Vinita Dubey, Soren Gantt, Robin Harrison, Kyla Hildebrand, Kristin Klein, Jesse Papenburg, Coleman Rotstein, Beate Sander, Susan Smith and Sarah Wilson); $\mathrm{NACl}$ liaison members (Lucie Marisa Bucci, Eliana Castillo, Amanda Cohn, Lorette Dupuis, Julie Emili, Deshayne Fell, Martin Lavoie,
Dorothy Moore, Monika Naus and Anne Pham-Huy); NACl ex-officio members (Vincent Beswick-Escanlar, Erin Henry, Mireille Lacroix, Marie Christine Lamontagne, Jennifer Pennock, Robert Pless, Guillaume Poliquin and Tom Wong); High Consequence Infectious Disease Working Group members (Caroline Quach [Chair], Shelley Deeks [Vice-Chair], YenGiang Bui, Kathleen Dooling, Robin Harrison, Kyla Hildebrand, Michelle Murti, Jesse Papenburg, Robert Pless, Beate Sander, Nathan Stall and Stephen Vaughan); and NACI Secretariat staff (Natalia Abraham, Paméla Doyon-Plourde, Veronica Ferrante, Nicole Forbes, Renee Goddard, Althea House, Shainoor Ismail, April Killikelly, Michelle Mathieu-Higgins, Austin Nam, Milan Patel, Angela Sinilaite, Matthew Tunis, Man Wah Yeung and Linlu Zhao) contributed to the development of the full guideline. The $\mathrm{NACl} C$ Chair, Vice-Chair and voting members gave final approval of the version of the full guideline to be published and agree to be accountable for all aspects of the work.

Funding: This work was supported by the Public Health Agency of Canada (PHAC). Funding for the National Advisory Committee on Immunization is provided by PHAC.

Content license: This is an Open Access article distributed in accordance with the terms of the Creative Commons Attribution (CC BY-NCND 4.0) license, which permits use, distribution and reproduction in any medium, provided that the original publication is properly cited, the use is non-commercial (i.e., research or educational use), and no modifications or adaptations are made. See: https://creativecommons.org/ licenses/by-nc-nd/4.0/

Acknowledgements: The authors thank the Alberta Research Centre for Health Evidence (Lisa Hartling, Jennifer Pillay and Aireen Wingert), for its evidence reviews that supported this guideline; the Public Health Agency of Canada and its collaborating provincial and territorial public health partners, for the COVID-19 epidemiology updates; and the stakeholders who provided feedback on the draft guideline, including the Canadian Immunization Committee, the Pan-Canadian Public Health Network's Special Advisory Committee on COVID-19, and the Special Advisory Committee's Technical Advisory Committee.

Correspondence to: National Advisory Committee on Immunization, phac.naci-ccni.aspc@canada.ca 